\title{
Abnahme und Wiederbefestigung der Suprakonstruktion bei Periimplantitis
}

\author{
Implantat-getragener Zahnersatz nimmt kontinuierlich zu. Der Bedarf an professioneller Reini- \\ gung dieses Zahnersatzes ebenfalls. Damit einher geht zeitversetzt allerdings auch ein weiterer \\ Trend - der zur manifesten Mukositis und Periimplantitis. Geeignete Therapien werden seit eini- \\ gen Jahren entwickelt. Gebührenrechtliche Einigkeit besteht in der Tatsache, dass die eigentliche \\ Periimplantitis-Therapie, zumindest in Form einer offenen chirurgischen Behandlung, weder in \\ der GOZ noch in der GOÄ beschrieben ist und folglich gemäß § 6 Abs. 1 auf dem Wege der Ent- \\ sprechungsberechnung in Ansatz zu bringen ist.
}

Weniger Einigkeit besteht indes bezüglich eines Teils der sogenannten Begleitleistungen, hier konkret zur Abnahme der Suprakonstruktion bei einer professionellen Belagentfernung oder einer Periimplantitis-Therapie. Zutreffende Leistungsbeschreibungen in der GOZ finden sich nicht, weder die Nr. 9050 (Entfernen/Wiedereinsetzen Aufbauelemente zweiphasiges Implantatsystem in der rekonstruktiven Phase) noch die Nr. 9060 GOZ (Auswechseln im Reparaturfall) spiegeln den tatsächlichen Leistungsinhalt wider. Bei einer Periimplantitis-Therapie ist primär nicht von einem solchen „Reparaturfall“ auszugehen, wenngleich dieser im Einzelfall durchaus hinzu treten könnte. Auch ist „Auswechseln“ der Austausch von Sekundärteilen des Implantates im Sinne von "alt gegen neu“, nicht genannt ist die bloße Wiederbefestigung eines Sekundärteils.

Die gemäß Leistungsbeschreibung in der Nr. 9060 nicht eingeschlossene „Abnahme und Wiederbefestigung der Suprakonstruktion" kann gegebenenfalls eine eigenständige zahnärztliche Leistung darstellen, die zusätzlich berechnet wird. Erfolgt zum Beispiel die nötige Abnahme einer Krone in Form einer aktiven „Entfernung“, beispielsweise durch Aufschlitzen, Hämmern oder Ultraschalllockerung gemäß Nr. 2290 „Entfernung einer Krone“, so ist in diesem Fall auch diese 2290 GOZ zutreffend berechnungsfähig. Zwangsläufig ist nach Wiederherstellung der Suprakonstruktion die daraufhin erforderliche Wie-

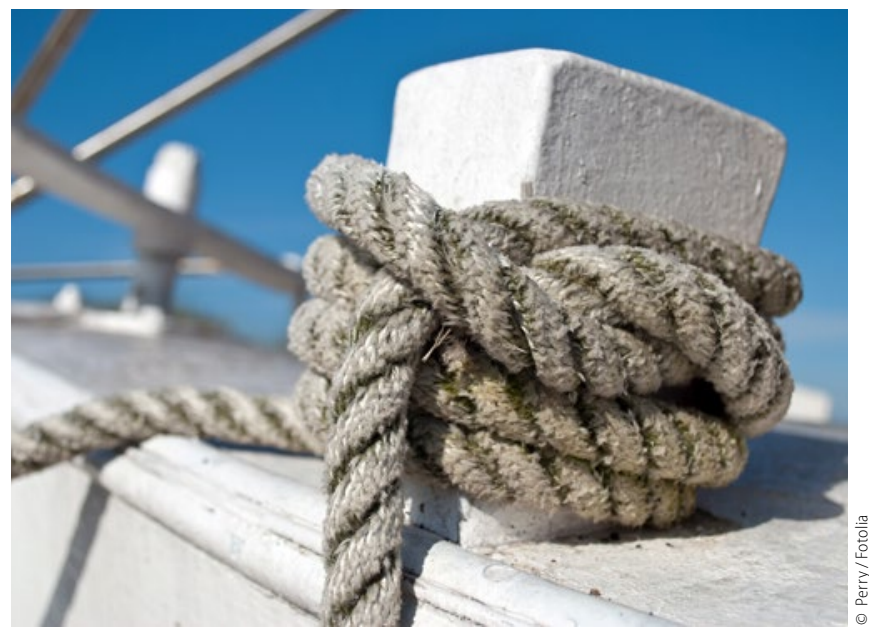

derherstellung und -eingliederung der Krone gemäß Nr. 2320 GOZ berechnungsfähig, dies auch im Fall einer Verblendreparatur, gegebenenfalls zuzüglich Wiedereingliederung der Brückenspanne nach Wiederherstellung gemäß 5110 GOZ.

Doch der oben beschriebene Fall einer Periimplantitis-Therapie ohne Reparaturbedarf für die bestehende Suprakonstruktion ist damit nicht zutreffend dargestellt. Wirft man einen Blick auf die aktuelle Kommentierung der Bundeszahnärztekammer, so lässt sich folgende Formulierung finden: „Wiederbefestigung der Aufbauelemente zum Zweck der Reinigung nach rekonstruktiver Phase ist nicht beschrieben und daher analog zu berechnen."

Das ist, wie erläutert, aus der Leistungsbeschreibung der Nr. 9060 GOZ abzuleiten, wie auch zuzüglich Gebührenziffern nach 2290, 2310, 2320 und 5110 GOZ belegen. Dennoch: Die Abnahme und Wiederbefestigung, das heißt die Demontage oder Remontage von bedingt abnehmbaren Sekundär- und Tertiärstrukturen wie beispielsweise verschraubte Stege als Suprakonstruktion auf Implantataufbauten ist nicht in der Gebührenordnung beschrieben. Genau dann ist die Analogie- oder Entsprechungsberechnung zutreffend.

In ALEX (www.alex-za.de) ist im Bereich „Entsprechungsliste“ eine Leistungsbeschreibung plus Analogziffer aufgeführt mit dem Wortlaut „Demontage, PZR der Implantat-Suprakonstruktion, Remontage, je Implantat“; zusätzlich gibt es eine Leistungsbeschreibung "Lappenoperation am Implantat zur Periimplantitisbehandlung“, die gegebenenfalls hinzukommt. Bekannt ist, dass zahnärztliche Leistungen, die auf Grundlage des $₫ 6$ Abs. 1 GOZ berechnet werden, alles andere als gesicherte Erstattung mit sich bringen.

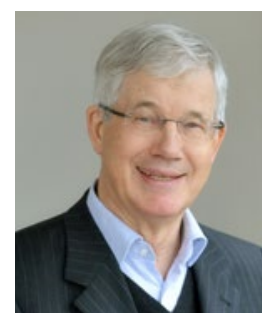

Dr. Peter H.G. Esser

GOZ-Experte und -Berater der ZA eG www.za-abrechnung.de 